\title{
Exploiting the " Rat Actinide Repository " at the Laboratory of RadioToxicology, CEA, France
}

\author{
Nina M. Griffiths ${ }^{1 *}$, Stéphanie Lamart ${ }^{1}$, Anne-Claire Humbert ${ }^{2}$, Anne Van der Meeren ${ }^{1}$ \\ ${ }^{1}$ Laboratoire de RadioToxicologie, CEA, Université Paris-Saclay, 91297 Arpajon, France \\ ${ }^{2}$ CEA, DAM, DIF, 91297 Arpajon, France
}

\section{Introduction}

The instigation of "registries" and "repositories" of biological samples from workers in the nuclear industry, both civil and military, provides an excellent source of data concerning the long-term effects of internal contamination by radionuclides (e;g. U.S. Transuranium and Uranium Registries, USTUR; Radiobiological Human Tissue Repository, RHTR). Similarly tissue archives exist concerning animal experiments. During many years of research dating from the 1960s at the Laboratory of RadioToxicology (LRT) a large number of tissue samples were collected from animals contaminated with alpha particleemitting actinides, mostly following inhalation. To date around 3000 samples and 7000 unused sections $(5 \mu \mathrm{m})$ from 1500 rats have been included in the inventory of samples at LRT. Samples are from a number of studies such as (i) pulmonary inflammatory responses and pathologies following inhalation of MOX [Mixed OXide, Uranium, plutonium $(\mathrm{Pu})$ ] oxide or Pu oxide, (ii) osteosarcomas following injection of soluble $\mathrm{Pu}$ and biokinetics and (iii) effects of different regimens for decorporation following contamination of wounds.

\section{Exploitation of samples from the repository: examples}

The formalin-fixed paraffin-embedded (FFPE) tissues have been mainly used for histopathological analyses and in particular for description of lung tumour types following inhalation of actinides (MOX, plutonium, neptunium). In addition inflammatory responses and fibrosis were studied in lungs from MOX-contaminated animals ${ }^{1}$. However these tissues may be used for other analytical purposes - such as identification of different elements or isotopes in particular tissue structures. For example, using lungs obtained from MOX-contaminated rats (Fig. 1), fibrotic areas containing aggregates of activity were identified by emulsion and solid track (CR39) autoradiography and dissected from sections (60 $\mu \mathrm{m}$ thick) and subjected to further analyses. The alpha spectrophotometry measurements show that the aggregates formed in the fibrotic tissue contain both $\mathrm{Pu}$ and $\mathrm{Am}$ and importantly, in the same expected isotopic/elemental ratios as calculated from isotopic decay of the particular MOX powder. Of note is the "in-growth" of Am. In animal experiments the time of contamination is known, the contaminant is known and so calculations of Am "in-growth" can be fairly exact. In the analysis of tissues from cases of accidental actinide contamination this may not necessarily be the case. It is important

*Corresponding author: nina.griffiths@cea.fr 
therefore to consider this when tissues are analysed many years after the actual contamination.

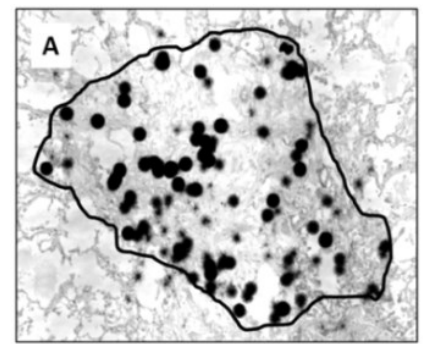

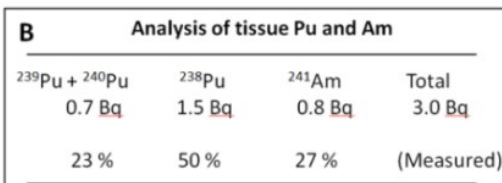

MOX Pu - Am at time of inhalation experiment (2004) $19.3 \% \quad 61.9 \% \quad 18.7 \% \quad$ (Calculated)

MOX Pu - Am at time of tissue analysis (2010) $\begin{array}{llll}17.8 \% & 54.5 \% \quad 27.6 \% \quad \text { (Calculated) }\end{array}$

Fig. 1. A: Autoradiograph (emulsion) of rat lung section with aggregates of activity in a fibrotic zone after inhalation of $\operatorname{MOX}(7,1 \% \mathrm{Pu})$. Counter staining haematoxylin-eosin. The delimited area was dissected using a microscope and fine needles. The tissue was weighed and analysed for $\mathrm{Pu}$ and Am following column separation and alpha spectrometry. B: Table showing measured Pu or Am activity in the dissected tissue and the different calculated isotopic fractions of MOX either at the time of inhalation (2004) or time of tissue analysis (2010).

More recent experiments using the iQID (ionizing-radiation Quantum Imaging Detector) camera where activity can be measured directly on a tissue section results showed that in lungs from MOX-contaminated rats, higher levels of activity were found in areas of fibrosis as compared with non-fibrotic regions ${ }^{2}$. This technique however has the advantage of being non-destructive and measures activity directly as opposed to dissection and lengthy destructive radiochemical procedures. To date however, differential isotopic analysis is not possible. Distinct lung pathologies (fibrosis, tumours) are consequential to inhalation of mainly insoluble actinides but following systemic administration of more soluble Pu forms osteosarcomas are the main result. Osteosarcoma FFPE samples from the LRT Rat Actinide Registry/Repository were analysed for different tumour-associated markers. The constitutive activation of the Wnt/beta-catenin pathway in these tumours was identified by nuclear and/or cytoplasmic localization of beta-catenin and a decrease in its inactive (phosphorylated) form in tissue sections ${ }^{3}$.

\section{Conclusions}

These tissues obtained from different contamination protocols with different actinides provide a source of material that may be exploited in a number of ways - histopathological analyses with new markers for example of proliferative lesions such as cancer-associated fibroblasts, activity measurements at a microscopic level by SIMS, comparisons of Am "ingrowth, nuclear forensic analyses of plutonium isotopes... . This tissue repository is a legacy for radiotoxicological measurements and also a significant resource for future that fulfil the remit of the 3 Rs (Replace, Reduce, Refine) for animal experimentation.

The authors are very grateful to Valérie Quéméneur for excellent organisational and cataloguing skills for the recent inventory of the tissue blocks and to the EU for financial support (MELODI).

\section{References}

1. N.M. Griffiths, A. Van der Meeren, P. Fritsch, M-C. Abram, J-F. Bernaudin, J.L. Poncy, Health Phys. 99(3): p. 347-56 (2010).

2. S. Lamart, B.W. Miller, A. Van der Meeren, A. Tazrart, J.F. Angulo, N.M. Griffiths, PLoS One. 12(10): 0186370 (2017).

3. K. Daino, N. Ugolin, S. Altmeyer-Morel, M-N. Guilly, S. Chevillard, Int J Cancer. 125(3): p. 612-20 (2009). 